\section{Novel causes of \\ Budd-Chiari-like \\ syndrome in two dogs}

\section{Harry Swales ${ }^{1}$, Elizabeth Bode ${ }^{1}$, Francisco Fernández Flores², Alistair Freeman ${ }^{1}$, Jeremy Mortier ${ }^{1}$, Matteo Rossanese ${ }^{1}$, Yi Lin Tan', Ranieri Verin' ${ }^{2}$, Erin O'Connell ${ }^{1}$}

1 University of Liverpool Small Animal Teaching Hospital, Liverpool, United Kingdom

2 University of Liverpool Veterinary Pathology, Liverpool, United Kingdom

\section{OBJECTIVES}

To outline the investigation of two unusual cases of BuddChiari-like syndrome.

\section{METHODS}

$\mathrm{N} / \mathrm{A}$

\section{RESULTS}

Case 1: A 6-year-old, male-neutered Brittany spaniel presented with a week's history of progressive ascites. Investigations revealed hypoalbuminaemia $(20 \mathrm{~g} / \mathrm{L})$ but normal globulins (26g/L). Resting bile acids $(5.6 \mu \mathrm{mol} / \mathrm{L})$, ammonia $(6 \mu \mathrm{mol} / \mathrm{L})$ and the UP:C (0.12) were within normal limits. Abdominocentesis yielded a high protein transudate. CT-angiography revealed progressive narrowing of the caudal vena cava ( $\mathrm{CaVC}$ ) at the level of the diaphragm but no evidence of intra- or extra-mural compression. No improvement was seen following caval stenting or after administration of human serum albumin or dexamethasone. Post-mortem examination revealed hepatic cirrhosis and a spherical appearance to the cranial liver resulting in extra-mural compression of the CaVC and hepatic veins. This was deemed the primary cause of the recurrent ascites.

Case 2: A 10-year-old, male neutered Border terrier presented with a four-day history of recurrent ascites. Biochemistry revealed hypoalbuminaemia $(21 \mathrm{~g} / \mathrm{L})$ and mild hypoglobulinaemia (24g/L). Post-prandial bile acids were not suggestive of a portosystemic shunt $(16.7 \mu \mathrm{mol} / \mathrm{L})$ and the UP:C (0.14) was within normal limits. Peritoneal fluid analysis was consistent with a high protein transudate. CT-angiography revealed focal stenosis of the CaVC caudal to the right atrium due to circumferential wall thickening. Post-mortem gross examination revealed mediastinal lymphadenomegaly, microscopically characterised by nodal histiocytic anthracosis, causing extra-mural compression of the CaVC. This abnormality was deemed the primary cause of the recurrent ascites.

\section{STATEMENT (CONCLUSIONS)}

These cases represent two previously unreported causes of Budd-Chiari-like syndrome in dogs, both caused by extra-mural compression of the CaVC.

\section{Fanconi-Syndrome in a Labrador Retriever}

\section{Corinna N. Weber ${ }^{1}$, Sabine Gerber-Andries ${ }^{1}$, Albert Schmidt ${ }^{2}$, Katrin Jaeger ${ }^{1}$, Elisabeth Mueller ${ }^{1}$}

1 Laboklin GmbH \& Co. KG, Bad Kissingen, Germany

2 Kleintierpraxis Graesser, Grossostheim, Germany

\section{OBJECTIVES}

Fanconi syndrome is a inadequate reabsorption in the proximal renal tubules because of congenital or acquired causes, resulting in a selective or generalized aminoaciduria and the loss of electrolytes, glucose, lactate and bicarbonate with the urine. Left untreated, Fanconi syndrome leads to progressive kidney failure.

\section{METHODS}

A male 11 year old Labrador Retriever was presented to the clinic with vomiting, lethargy, weakness, and polydipsia. Increased levels of urea and creatinine were found in the blood, as well as a mild anemia. Urinalysis showed a severe aminoaciduria and glucosuria.

\section{RESULTS}

The findings of the clinical examination together with blood work and urinalysis were consistent with Fanconi syndrome. In the following months, the general condition deteriorated, and serum concentrations of urea and creatinine increased. The dog was euthanized 17 months after the first presentation. Both kidneys showed a highly uneven surface of firm consistency and massive concretion deposits in the renal pelvis. Pathohistological examination revealed a bilateral severe chronic interstitial lymphoplasmacellular pyelonephritis with severe nephrolithiasis and cyst formation. The concrements found in the renal pelvis were beige and smooth and mostly composed of struvite together with calcium-rich apatite.

\section{STATEMENT (CONCLUSIONS)}

For Labrador Retrievers, several cases of Fanconi syndrome have been reported in the literature, in some cases linked to a copper-mediated hepatitis. In the present case, no copper storage disease was detected, and there was no evidence of alimentary or drug related reasons. Further research is needed to identify possible congenital causes in the Labrador Retriever. 